\title{
Protection de la nappe de Lyon contre la pollution
}

\author{
par J.-F. Busse \\ Ingénieur Hydrogéologue, \\ Agence de Bassin Rhône-Méditerranée-Corse
}

Les plaines alluviales constituent des lieux privilégiés pour l'alimentation en eau potable. Elles sont également particulièrement propices au développement urbain et industriel ainsi qu'à l'implantation des voies de communication, tous aménagements qui font peser des risques de pollution sur la nappe.

En raison de l'importance des nappes alluviales pour l'alimentation humaine, l'Agence de Bassin RhôneMéditerranée-Corse avait inscrit à son premier programme d'intervention la réalisation d'études, en vue de promouvoir les dispositions les meilleures pour assurer la protection des zones de captages dans ces nappes. Ce but implique d'avoir une bonne connaissance des pollutions existantes et d'être renseigné sur le mécanisme de leur propagation et de leur évolution.

Le domaine d'application retenu pour ces études a été la plaine alhuviale de l'Est Lyonnais qui comporte des zones de captages devant alimenter 1 million d'habitants.

\section{Cadre géographique}

La région étudiée, d'une superficie de $280 \mathrm{~km}^{2}$, correspond à la plaine alluviale du Rhône entre le confluent de l'Ain et Lyon, ainsi qu'aux plaines adjacentes en rive gauche. (Voir figure 1.)

Elle est bien délimitée piézométriquement et est assez vaste pour présenter plusieurs zones industrielles, urbaines et agricoles, sur différentes sortes de terrains alluviaux: alluvions modernes, couloirs fluvio-glaciaires et moraines glaciaires.

Dans les limites de cette zone, existe une population de plus de 100000 habitants dont plus du tiers n'est pas raccordé à des réseaux d'assainissement. Trois cent-cinquante établissements industriels y sont également implantés et peu sont desservis par un réseau d'égouts.

\section{Généralités sur le programme et les méthodes de recherche utilisés}

En 1967, nous avons effectué un bilan des connaissances acquises sur la plaine de Lyon et établi un programme de travail. Ce dernier a été entrepris en liaison avec les administrations concernées (O.R.E.A.M., Directions Départementales de l'Agriculture et de l'Equipement, Service de la Navigation, Communauté Urbaine).

Il s'agissait de compléter les connaissances déjà acquises par les travaux antérieurs du Bureau de Recherches Géologiques et Minières et de la Faculté des Sciences de Lyon sur le réservoir. Une recherche exhaustive des risques de pollution était ensuite prévue ainsi qu'une analyse de la qualité actuelle des eaux de la nappe. Enfin, une recherche des mécanismes de propagation de la pollution devait être entreprise.

Aux organismes déjà cités, ont été associés pour élaborer ou développer certains points du programme, ainsi que pour réaliser les travaux de terrain:

- le Service Géologique d'Alsace-Lorraine (S.G.A.L.),

- la Compagnie de Prospection Géophysique Française,

- le Centre d'Etudes Nucléaires de Grenoble (C.E.N.G.),

- l'Association pour la Recherche et le Développement des Méthodes et Processus Industriels (Ecole Nationale Supérieure des Mines de Paris-A.R.M.I.N.E.S.), - la Société Anonyme Française d'Etudes et de Gestion,

- la Compagnie Générale des Eaux,

- la Société Lyonnaise des Eaux,

- la Compagnie Générale de Géophysique,

- la Béarnaise, SONDARALP.

Les analyses chimiques ont été effectuées par l'Institut Pasteur de Lyon. 


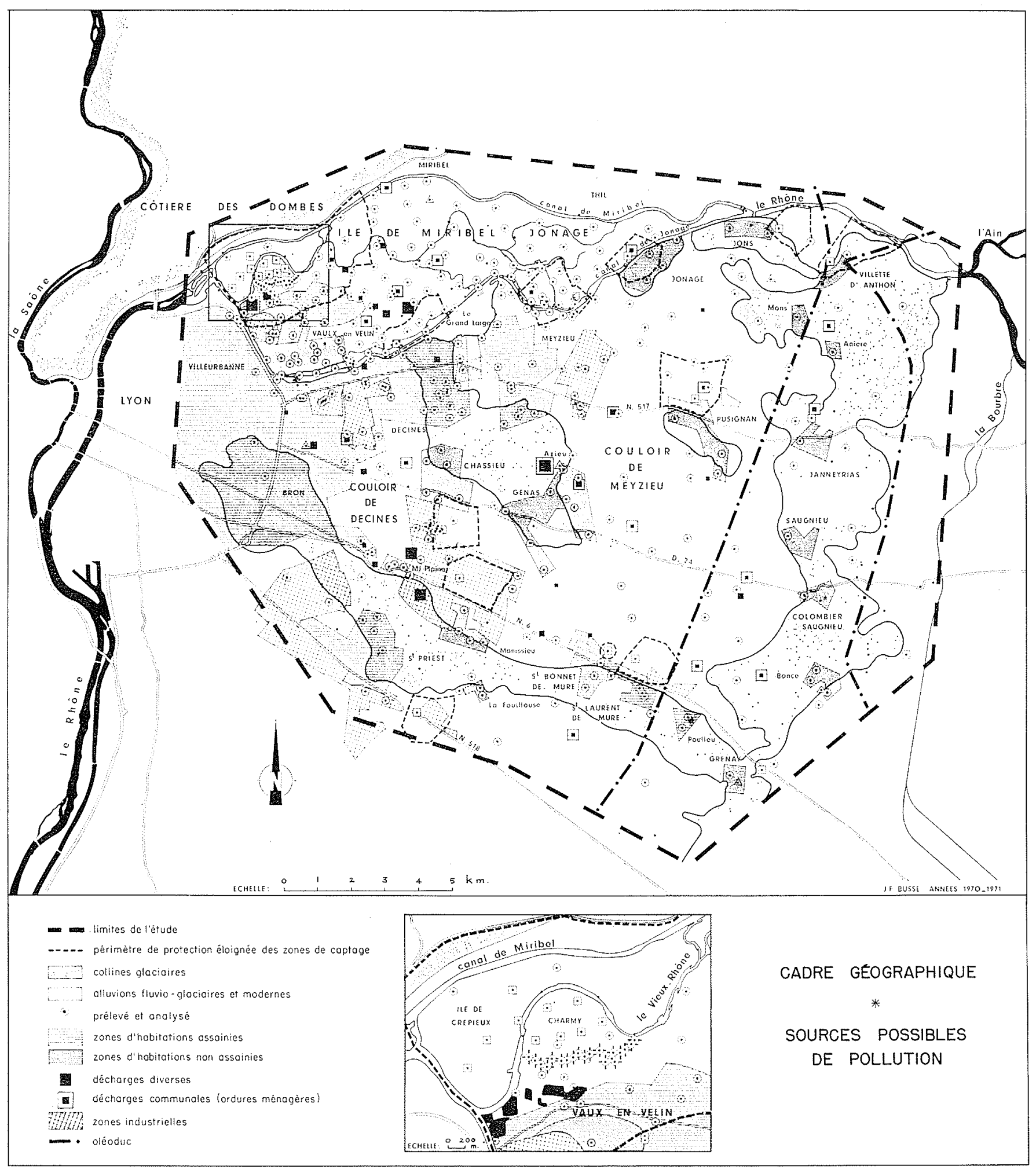


Il a été fait un large usage de la géophysique pour rechercher les écrans argileux protecteurs dans la zone non saturée et pour dresser la cante des perméabilités dans les alluvions saturées (par corrélation entre les données d'essais de pompage et le «facteur de formation" des mêmes sites).

Certaines méthodes ont été mises au point à l'occasion de cette étude :

- l'usage des traceurs radioactifs pour mesurer in situ la vitesse de transit en zone non saturée (C.E.N.G.);

- la prospection électrique du déplacement d'une saumure injectée sur puits unique, pour calculer la dispersion (ainsi que la direction et la vitesse d'écoulement), (A.R.M.I.N.E.S. et S.G.A.L.). Cette méthode a été retenue après un essai simultané avec d'autres procédés tels que traceurs radioactifs naturels ou artificiels et traceurs chimiques, en puits unique ou multiple.

Enfin, une recherche technologique en matière de sondages a permis la création, au moindre coût, du procédé "multitubes», pour connaitre sélectivement la qualité de la nappe à différentes profondeurs d'une même verticale.

\section{Connaissance du milieu}

Elle avait pour but la détermination de la vulnérabilité de la nappe.

Le complément aux études hydrogéologiques déjà entreprises a porté sur la nature du réservoir aquifère et le comportement de la surface piézométrique.

\section{Le réservoir aq̨uifère}

La géométrie du réservoir aquifère et ses caractéristiques ont été étudiées à l'aide de 1000 sondages électriques, étalonnées sur 145 sondages mécaniques (dont une quarantaine exécutés à l'occasion de l'étude) et 105 essais de pompage.

L'ossature molassique est généralisée sous les collines glaciaires, ce qui confirme l'hypothèse du Professeur David. Les dépôts d'argile morainique tapissent le fond des couloirs fluvio-glaciaires et de la moitié occidentale des alluvions modernes. Ces changements de nature du substratum entraînent des variations de perméabilité. Celle de l'argile morainique est de $10^{-9} \mathrm{~m} / \mathrm{s}$ environ. La molasse est, par contre, relativement perméable : $10^{-4} \mathrm{~m} / \mathrm{s}$, selon les essais de pompage pratiqués sur plusieurs dizaines de mètres (J.-J. Collin, 1971). On ne peut considérer la molasse comme un substratum imperméable par rapport aux alluvions modernes et encore moins vis-à-vis des dépôts glaciaires. Les alluvions fluvio-glaciaires sont nettement plus perméables $\left(710^{-3} \mathrm{~m} / \mathrm{s}\right.$ en moyenne) que les alluvions modernes $\left(210^{-3} \mathrm{~m} / \mathrm{s}\right.$ en moyenne). Les dépôts glaciaires sont, dans l'ensemble, très peu perméables $\left(10^{-5} \mathrm{~m} / \mathrm{s}\right)$.

Cette étude a permis d'établir une carte des perméabilités.

\section{La surface piézométrique}

Pour étudier le comportement de la surface piézométrique, aux 250 points d'observation préexistants, ont été ajoutés 80 piézomètres et 45 échelles limnimétriques; 200 points d'eau nouveaux ont, en outre, été inventoriés dans les couloirs fluvio-glaciaires et les collines glaciaires pour étudier le raccordement entre ces formations. Quatre campagnes piézométriques générales ont été effectuées sur tous ces points de mai 1970 à septembre 1971 (voir figure 2).

Les conclusions de cette étude sont les suivantes:

- les limites de la nappe étudiée sont constituées par les crêtes phréatiques des collines glaciaires; ces crêtes isolent la zone étudiée de l'aquifère de la Bourbre (à l'Est) et de celui du couloir d'Heyrieux (au Sud) : la séparation piézométrique totale entre les couloirs fluvio-glaciaires de Meyzien et Décines empêche tout passage polluant de l'un à l'autre;

- sous l'aquifère glaciaire, existe une nappe molassique qui peut être contaminée par sa voisine morainique;

- l'allure générale de la surface piézométrique (direction et pente) est tributaire de la forme du substratum, qui détermine «nappes en dôme » et axes drainants;

- les eaux souterraines issues du glaciaire (et pouvant véhiculer des pollutions urbaines) s'écoulent dans les alluvions fluvio-glaciaires (où elles peuvent rencontrer d'éventuelles pollutions industrielles), puis gagnent l'aquifère moderne (et de possibles pollutions issues de diverses décharges et de l'agglomération de Vaulxen-Velin);

- le passage problématique des eaux fluvio-glaciaires de Decines dans les alluvions modemes est lié à l'étude des infiltrations possibles de la Rize qui ne semble toutefois pas jouer un rôle efficace de barrière hydraulique;

- sur leur trajet, les eaux souterraines rencontrent plusieurs zones de captage communales, avant de converger, pour la plupart, vers celles de la Communauté Urbaine de Lyon; l'alimentation en eau potable de la région lyonnaise est donc tributaire de la qualité de l'ensemble de la nappe étudiée.

\section{Vulnérabilité de la nappe}

La propagation de la pollution dans la zone non saturée rencontre de nombreux obstacles naturels dans les terrains glaciaires, très épais (10 à $40 \mathrm{~m}$ ) et qui comportent partout, soit une couverture argileuse, soit des intercalations de même nature très importantes $(5$ à $10 \mathrm{~m})$. La partie amont des couloirs fluvio-glaciaires présente les mêmes intercalations importantes, les dépôts superficiels étant peu épais et plus ou moins perméables. Le reste des couloirs ainsi que les alluvions modernes sont mal protégés contre la propagation de la pollution dans la zone non saturée. Le rôle de tampon, que constituent les fortes épaisseurs non saturées, joue surtout en faveur des terrains déjà favorisés par la présence d'écrans argileux: le glaciaire et la partie amont des couloirs fluvioglaciaires.

L'aquifère fluvio-glaciaire s'avère encore plus vulnérable que celui des alluvions modernes. Par contre, l'aquifère glaciaire est relativement protégé contre la propagation de la pollution. 


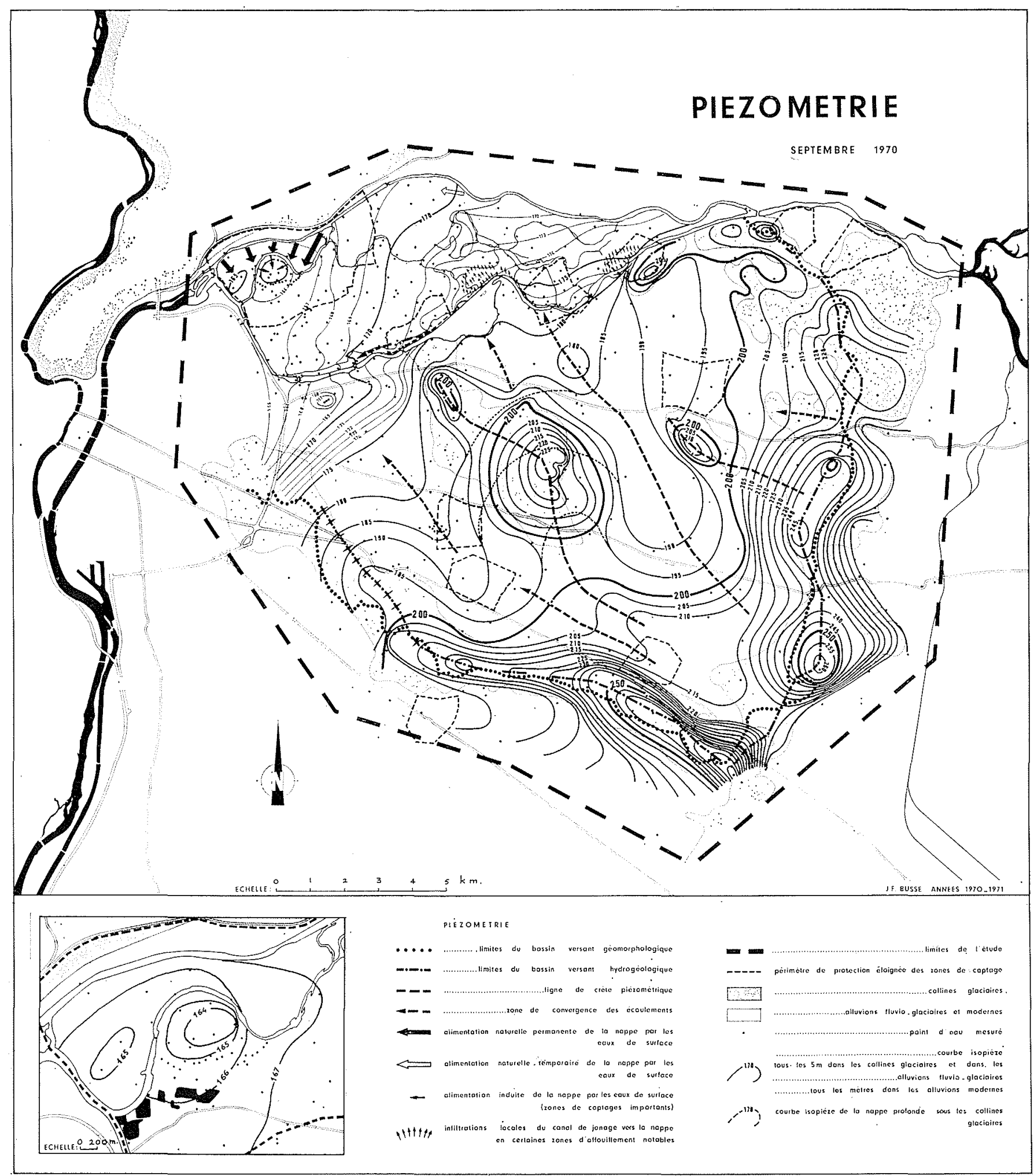




\section{Inventaire des sources de pollution}

Cet inventaire a permis de connaitre les points de la nappe les plus menacés (voir figure 1).

Sur 355 établissements industriels recensés, 80 ont fait l'objet d'enquêtes (dont une quarantaine très détaillées avec inventaire des puits perdus et de la nature des eaux rejetées). Ce travail a permis d'établir une carte des risques de pollution chronique ou accidentelle.

Les réseaux d'assainissement ont été recensés et leurs caractéristiques générales ont été reportées sur une carte en même temps que tous les renseignements concernant les types d'assainissement et de rejets urbains. Dans les 16 communes de la région étudiée (115000 habitants), 40000 habitants ne sont pas encore raccordés à des réseaux d'assainissement qui sont, en de nombreux endroits, inexistants.

Une grande partie des gravières existant dans la plaine de Lyon sont transformées en décharges publiques ou clandestines. Les plus importantes ont été reportées sur une carte: 140 ont été recensées, 35 étudiées plus spécialement.

\section{Étude générale sur la qualité de l'eau}

Cette étude a mis en ouvre:

- deux campagnes généralos de prélèvements et d'analyses en mai et septembre 1970, sur 400 points d'eau souterraine et une trentaine de points d'eau de surface;

- deux campagnes plus restreintes sur une centaine de points, choisis d'après les résuitats des campagnes précédentes en mai et septembre 1971;

- une campagne en octobre 1972 sur ces cent points, augmentés de 150 en rive droite du canal de Miribel, non comprise jusque là dans les observations.

Afin d'assurer une bonne représentativité des échantillons analysés, les prélèvements d'eau souterranine sur les puits hors service ont été faits après un pompage d'au moins 1,5 à 2 fois le volume d'eau initial dans l'ouvrage, cette procédure ayant fait au préalable l'objet d'essai de représentativité. Les échantillons prélevés étaient remis le soir même à l'Institut Pasteur de Lyon.

\section{Résultats des analyses}

L'analyse des éléments minéraux fondamentaux a été faite sur l'ensemble des points. Des analyses spéciales concernant la bactériologie et des éléments toxiques ou indésirables ont porté sur un nombre de points plus limités choisis en fonction de leur proximité des sources possibles de pollution.

Les résultats des analyses ont été reportés sur 14 cartes qui rendent compte des principaux éléments analysés:

Sur l'ensemble des points:

- les conductivités, les T.A.C. (titres alcalimétriques complets), les chlorures $\left(\mathrm{Cl}^{-}\right)$, les sulfates $\left(\mathrm{SO}_{4}^{-}{ }^{-}\right)$, les nitrates $\left(\mathrm{NO}_{3}{ }^{-}\right)$, les T.H. (titres Hydrotimétriques), les ions sodium $\left(\mathrm{Na}^{+}\right)$, les ions potassium $(\mathrm{K}+)$.
Sur plus de la moitié des points:

- les coliformes.

Sur $80 \%$ des points:

- les détergents.

Sur $60 \%$ des points:

- les hydrocarbures.

Sur une cinquantaine de points:

- le chrome, le phénol, le cyanure.

Sur une vingtaine de points:

- les toxiques et indésirables: fluor, zinc, fer, manganèse.

Nous commenterons successivement huit de ces cartes qui correspondent toutes à la campagne de prélèvement de mai, sauf pour les détergents pour lesquels il s'agit de la campagne de septembre. Les variations éventuelles d'une campagne à l'autre sont évoquées dans le commentaire.

\section{Les conductivités}

- La mesure de la conductivité permet d'évaluer rapidement mais approximativement la minéralisation globale d'une eau potable.

- Les eaux de la nappe de Lyon ont une minéralisation moyenne (400 à 600 micromho. $\mathrm{cm}^{-1}$ ) sur l'ensemble de la plaine. Cependant, au voisinage du Rhône (canal de Miribel et canal de Jonage), la conductivité descend endessous de 400 micromho. $\mathrm{cm}^{-1}$, indiquant ainsi un pas. sage des eaux superficielles (270 à 300 mioromho. $\mathrm{cm}^{-1}$ ) dans les eaux souterraines. Cette influence est surtout sensible en trois points:

- l'extrémité amont de l'île de Miribel-Jonage;

- le long du canal de Jonage, à partir du pont de Meyzieu notamment, ce qui prouve le caractère relatif de l'étanchéité du fond du canal, dont la ligne d'eau est en charge par rapport à la nappe;

- l'extrémité occidentale des alluvions modernes de l'île avec notamment les zones de captage de Crépieux et de Charmy.

- Des minéralisations excessives (supérieures à $1000 \mathrm{mi}$ cromho. $\mathrm{cm}^{-1}$ et très importantes (de 800 à $1000 \mathrm{mi}$ cromho. $\mathrm{cm}^{-1}$ ) sont réparties en une trentaine de points sur l'ensemble de la nappe. Elles figurent notamment:

- à l'intérieur des périmètres de protection de Charmy où elles semblent liées à des décharges dont certaines situées en bordure du périmètre de protection immédiate;

- à l'aval de la décharge de la Ville de Lyon, à Genas (Azieu);

- dans les zones urbanisées ainsi que de nombreuses fermes.

\section{Les chlorures}

- En France, il apparait comme souhaitable de ne pas dépasser $250 \mathrm{mg} / 1$ de chlorures. 
- Sur la carte des chlorures, les teneurs des eaux souterraines et des eaux de surface ne se différencient pas. Un seul point présente une teneur importante $(440 \mathrm{mg} / \mathrm{l})$ et une vingtaine de points dépassent $50 \mathrm{mg} / 1$.

- La nappe sous Vaulx-en-Velin est caractérisée par une langue de teneur plus élevée qui s'étend du centre de l'agglomération jusqu'à la barrière de contrôle de Charmy.

\section{Les sulfates}

- Selon les normes françaises, les concentrations supérieures à $250 \mathrm{mg} / 1$ sont à éviter.

- L'influence des eaux superficielles plus chargées en sulfates est nettement visible; on passe de $10-20 \mathrm{mg} / 1$ à 20-30 mg/l à l'aval du canal de Jonage.

- La large bande exceptionnelle que nors avons déjà observée à Vaulx-en-Velin est ici précisée par plusieurs gammes successives de teneurs croissantes.

La pollution prend naissance $2 \mathrm{~km}$ à l'amont de l'agglomération, dans des zones d'ordures; elle s'accroît ensuite au niveau de la localité et augmente encore avec les décharges situées dans le périmètre de protection de Charmy et atteint un maximum dans cette zone de captage. Cette dernière est cependant peu touchée pour les raisons expliquées ci-dessus (voir les "Résistivités").

\section{Les nitrates}

- Il est recommandé de tenir compte pour l'eau potable en France, du fait qu'une teneur en nitrates supérieure à $44 \mathrm{mg} / 1$ est susceptible de provoquer des troubles, notamment chez les nourrissons.

- La carte traduit nettement l'influence des infiltrations du canal de Jonage dans les alluvions modernes.

Les teneurs en nitrates pour l'ensemble de la nappe sont beaucoup plus différenciées que pour les autres éléments, notamment dans les terrains glaciaires et fluvioglaciaires. Les différences de nature géologique entre ces deux types de terrains ne suffisent pas à expliquer de telles disparités.

- La diversité des teneurs pourrait coïncider avec certaines inégalités d'emploi des engrais. Il serait intéressant de préciser ce point par une enquête car nous n'avons pas d'éléments nous permettant d'affirmer cette coïncidence qui nous est simplement suggérée par des différences d'utilisation des sols. Dans l'île de Miribel-Jonage, les concentrations sont, dans l'ensemble, plus faibles (influence des eaux de surface), la zone de Vaulx-en-Velin faisant toujours exception.

Les points présentant une teneur supérieure à $44 \mathrm{mg} / 1$ sont au nombre d'une centaine, répartis sur toute la plaine.

\section{Les titres hydrotimétriques}

- Il est souhaitable que les eaux potables présentent un T. H. inférieur à 30 degrés français.

- La carte des T.H. rend particulièrement bien compte de l'évolution de la qualité de la nappe $\left(25\right.$ à $\left.30^{\circ}\right)$ au contact des infiltrations d'eaux superficielles (canal de Jonage : 15 à $17^{\circ}$ ) qui abaissent les teneurs d'eau souterraine à 20-25 dans les alluvions de l'île.
L'origine des cours d'eau de cette île est aussi mise en lumière. Dans la partie amont, les contre-canaux de la Rize et du Gua drainent une eau surtout rhodanienne. Dans le Rizan et les lônes du Vieux-Rhône, l'eau est celle de la nappe des alluvions modernes. Au débouché de la brèche de Neyron, l'eau du Rhône prédomine à nouveau.

- Les T. H. dépassent $30^{\circ}$ en de très nombreux points, surtout dans la partie occidentale de l'île, ainsi qu'à la décharge de la Ville de Lyon (à Azieu).

\section{Les détergents}

La campagne de septembre nous a paru plus significative en raison d'erreurs systématiques qui semblent s'être produites lors des manipulations en mai.

- Aucune norme de potabilité n'a été prévue par la législation française pour les détergents. L'Organisation Mondiale de la Santé demande que les teneurs exprimées en ABS n'excèdent pas $0,500 \mathrm{mg} / 1$.

- La majorité des points analysés dans la plaine de Lyon a des concentrations très faibles n'excédant pas $0,020 \mathrm{mg} / 1$.

Les teneurs excessives et importantes sont seulement au nombre de 5. Elles sont toutes situées dans des zones urbanisées ou à l'intérieur de fermes.

- Toutes les zones de captage présentent de très faibles concentrations (inférieures à $0,020 \mathrm{mg} / 1$ ).

\section{Les hydrocarbures}

- Les normes des U.S.A. indiquent qu'au-dessus d'une teneur de $1 \mathrm{mg} / 1$ de pétrole raffiné, l'eau est impropre à la consommation. La réglementation française exige l'absence d'odeurs ou de mauvais goûts.

- L'ensemble de la nappe est net d'hydrocarbures: une quarantaine de points sur 250 présentent des traces inférieures au seuil de sensibilité analytique.

\section{Les toxiques et indésirables}

Les teneurs en arsenic, cadmium, plomb, cuivre, fluorures, zinc, fer et manganèse sont très faibles et toujours conformes aux normes de potabilité de la législation française. Les teneurs en phénols et cyanures sont trop proches du seuil de sensibilité analytique pour être interprétables. Des concentrations en chrome de 0,040 à $0,060 \mathrm{mg} / \mathrm{l}$ ont été observées en mai 1970 , sur quatre points, en bordure du canal de Jonage; la teneur de $0,020 \mathrm{mg} / 1 \mathrm{n}$ 'a jamais été dépassée en septembre 1970.

\section{Essai de synthèse et d'interprétation}

Les campagnes d'analyses ont aussi permis de mettre en évidence divers faciès sans doute caractéristiques de certains types de pollution.

Tous les résultats d'analyses chimiques ont été reportés sous forme de diagrammes Shoeller-Berkaloff.

Les eaux souternaines étudiées sont du type bicarbonaté calcique (lié aux éléments calcaires d'origine jurassique et crétacée), avec des teneurs faibles à très faibles en $\mathrm{Mg}, \mathrm{Na}, \mathrm{K}, \mathrm{Cl}$ et $\mathrm{SO}_{4}$. Les effets de la pollution seront particulièrement visibles sur ces ions (voir figure 3 ). 


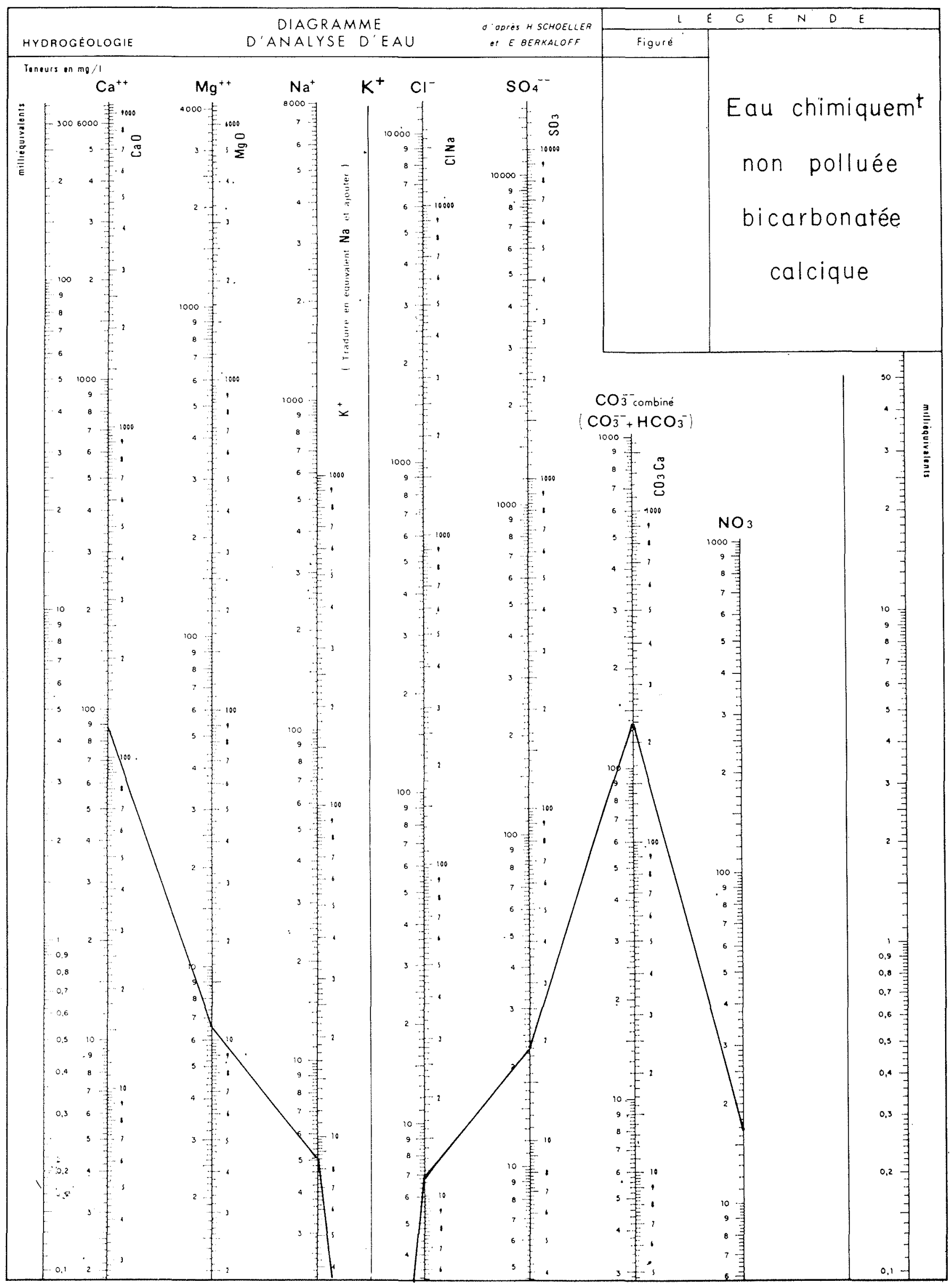


Une analyse des diagrammes nous a permis de dégager différentes familles de courbes auxquelles nous avons rattaché les graphiques présentant une allure identique. Dans chacune de ces familles, nous avons choisi un diagramme qui nous a paru particulièrement caractéristique de cet ensemble. Trois diagrammes caractéristiques sont bien marqués (voir figure 4):

- un diagramme de type chloruré sodique,

- un diagramme de type chloruré potassique,

- un diagramme de type sulfaté calcique.

Les points d'eau dont les diagrammes peuvent être rattachés à une de ces trois courbes caractéristiques ont été marqués sur une carte avec une couleur particulière à chaque type. Nous avons regroupé, sous une quatrième couleur, divers autres types de pollution.

Compte tenu du sens de l'écoulement des eaux souterraines, nous avons tenté de corréler ces différents diagrammes caractéristiques avec les diverses sources de pollution inventoriées. Sur les effluents de ces dernières, nous avons effectué des analyses dont les résultats ont été répertoriés sous forme de diagramme. La comparaison entre ceux-ci et les diagrammes caractéristiques nous a permis d'émettre les hypothèses suivantes:

- le diagramme des eaux vannes et des décharges à prédominance d'ordures ménagères s'apparenterait à la famille de type chloruré sodique;

- le diagramme des effluents de stabulation (purin) s'apparenterait à la famille chlorurée potassique;

- le diagramme des décharges à prédominance de déchets de construction s'apparenterait à la famille de type sulfaté calcique.

\section{Propagation de la pollution}

Parallèlement aux différents constats sur les caractéristiques de la nappe, la qualité de ses eaux et les risques de pollution, une étude sur les conditions de propagation des polluants a été entreprise après un examen bibliographique des connaissances acquises en France et à l'étranger.

\section{Etude bibliographique}

En 1969 et 1970, le Service Géologique d'Alsace-Lorraine a effectué une recherche bibliographique très importante dont nous avons pu bénéficier et que nous avons, en 1972, fait actualiser et compléter par le même service.

La recherche avait porté sur les polluants, la pollution des eaux souterraines et sa propagation. Le complément devait surtout envisager les techniques d'études de la migration des polluants.

L'étude des mécanismes de propagation en milieu non saturé a surtout porté sur les phénomènes d'appanvrissement: filtration, précipitation, absorption physique, chimisorption, échanges d'ions, dégradation biologique et rétention capillaire. En milieu saturé, les études ont surtout porté sur la direction et la vitesse de propagation et à un degré moindre sur les phénomènes de dispersion.

L'étude de la pollution par les ions minéraux fondamentaux a été faite surtout pour la contamination d'origine urbaine et celle due aux décharges d’ordures ménagères. Celles-ci sont particulièrement polluantes lorsqu'elles sont en contact direct avec la nappe. Cette dernière présente alors trois zones successives: réduction, transition, oxydation.

La pollution diminue avec le temps.

L'étude de la pollution par les substances toxiques et indésirables a été peu poussée et ne porte que sur quelques cas particuliers.

La propagation de la pollution biologique a été bien étudiée. En milieu non saturé, les virus sont fixés moins facilement que les bactéries. La fixation est diautant plus importante que la granulométrie est plus fine et le terrain plus argileux. En milieu saturé, la pollution biologique suit toujours le sens des filets liquides. A la longue, on observe la formation d'un lit bactérien qui freine et peut même arrêter la migration des bactéries. Des études donnent des distances de propagation de 30 à $360 \mathrm{~m}$ pour les virus, au lieu de 20 à $120 \mathrm{~m}$ pour les bactéries.

L'étude de la pollution par les hydrocarbures a été poussée assez loin. En zone non saturée, l'essence se propage deux à seize fois plus vite que l'eau dans un ternain de granulométrie très fine. Les hydrocarbures peuvent se propager moins vite que l'eau dans les sols à plus forte granulométrie.

En zone saturée, la propagation se fait au niveau de la frange capillaire jusqu'à l'obtention d'une position d'équilibre en forme de lentille (de quelques dizaines de mètres). Mais les éléments solubles libérés pouvent se propager sur plusieurs centaines de mètres, voire sur plusieurs kilomètres. En cas de pollution accidentelle, l'intervention doit être le plus rapide possible, pour tenter d'excaver les terrains pollués avant que les hydrocarbures n'atteignent la nappe. Si cela n'est pas possible, il reste le pompage, l'absorption ou le brûlage de ce qui flotte à la surface de la nappe. En cas de pollution chronique, la prévention doit intervenir à tous les stades de l'extraction, du traitement, de la distribution et de l'utilisation.

La pollution par les détergents peut être amenuisée en zone non saturée par l'absorption (terrains argileux) et la dégradation biologique. En zone non saturée, les détergents peuvent se déplacer sur plusieurs kilomètres.

Les pesticides, en zone non saturée, semblent facilement fixés par la couche supérieure de terrain; mais les plus courants (organo-chlorés) ne sont pratiquement pas dégradables. En zone saturée, l'absorption est faible. Les pesticides sont dans l'ensemble moins difficilement dégradés en milieu anaérobie.

Sur les substances radioactives, la zone non saturée peut jouer un rôle fixateur. La protection porte sur le stockage des déchets et le traitement des eaux polluées.

\section{Expérimentation ef recherche}

A l'occasion des campagnes de prélèvements ou à partir d'une expérimentation plus ou moins élaborée, nous avons essayé de retrouver les résultats des études bibliographiques, d'observer les conditions particulières de propagation de la pollution dans la plaine de Lyon et de mettre au point des méthodes de prévision de la propagation. Les interprétations de ces recherches ne sont pas encore achevées et certaines sont encore à étayer par des applications pratiques. Nous rendons compte ci-après des résultats obtenus jusqu'ici. 


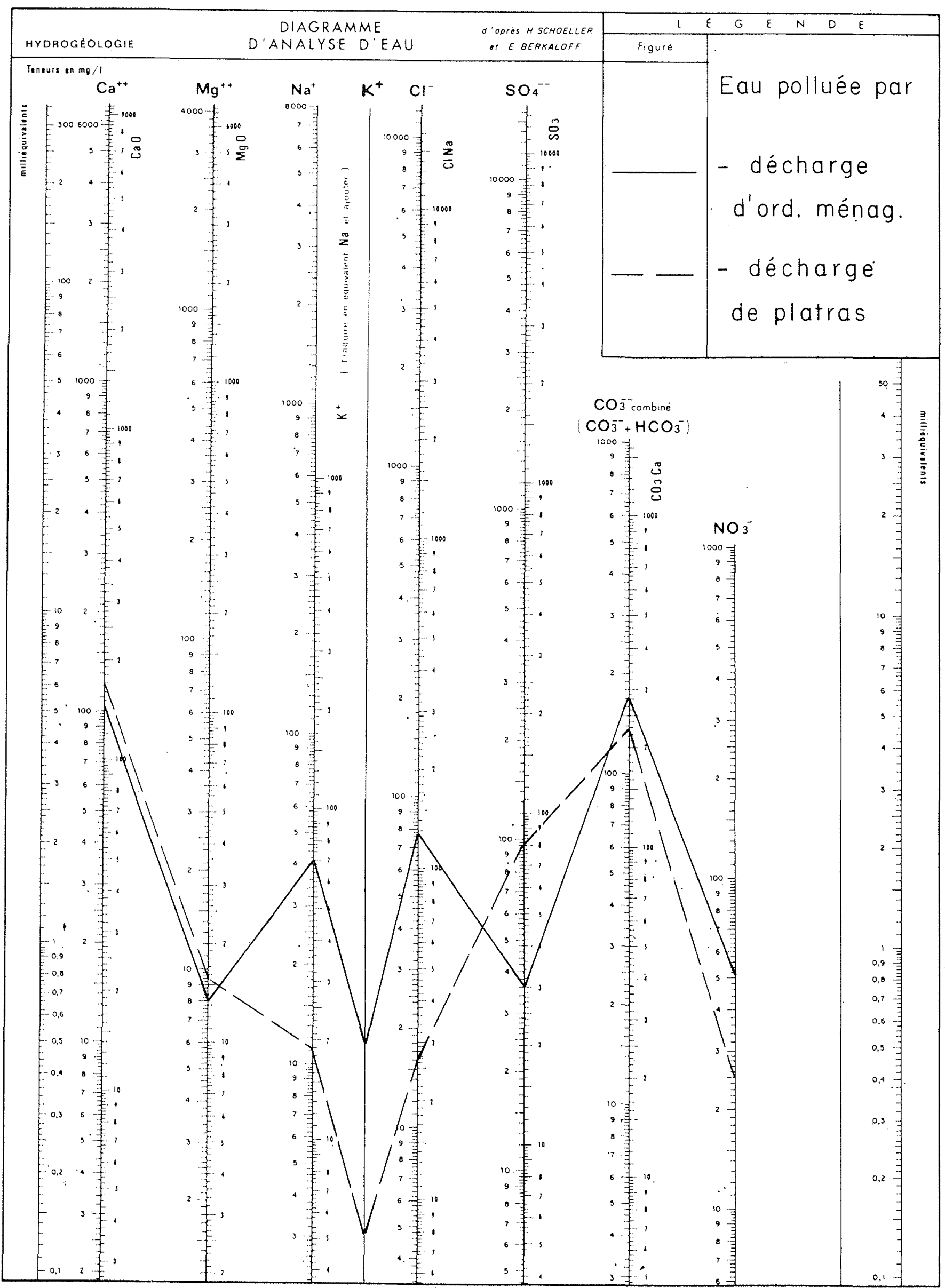




\section{Etudes et expérimentations locales}

- Mise en évidence de phénomènes de propagation de la pollution sur l'ensemble de la nappe.

Les teneurs observées localement lors des campagnes de mesures ont été utilisées comme traceurs naturels pour étudier la propagation de la pollution et son évolution.

La propagation de la pollution apparaît vraisemblable à l'aval de plusieurs points et notamment de la décharge de la Ville de Lyon à Azieu, de la zone de décharges au sud de Charmy.

Il semble que la pollution observée dans les couloirs fluvio-glaciaires ne réapparaisse pratiquement pas dans les alluvions modernes de l'île de Miribel-Jonage. Une hypothèse avancée est que les probables infiltrations faiblement minéralisées du canal de Jonage vers la nappe diluent fortement les pollutions venues de l'aquifère fluvio-glaciaire. Ces infiltrations se produisent essentiellement au droit des surcreusements constatés autour des ouvrages qui traversent le canal. On trouve, à l'aval du canal de Jonage, des eaux dont le diagramme s'apparente davantage au Rhône qu'aux diagrammes observés à l'amont dans les couloirs fluvio-glaciaires (voir figure 5).

Il semble que les eaux souterraines soient en voie de contamination dans la moitié occidentale de l'ile de Miribel-Jonage. Les causes de cette dégradation seraient à chercher dans les rejets domestiques et surtout dans les zones de décharges situées à l'est de Vaulx-en-Velin et à proximité de Charmy. Il est nécessaire de suivre l'évolution de la qualité de l'eau dans ce secteur ainsi que dans les couloirs fluvio-glaciaires.

La pollution est déjà observable dans la barrière de multitubes qui contrôlent la qualité de l'eau souterraine à l'amont des pulits de cette zone de captage.

La répartition verticale de la pollution sur toute l'épaisseur de la nappe est étudiée à partir des analyses effectuées sur une ligne de 20 "multitubes» (4 niveaux de prélèvements par multitube), échelonnés sur $1400 \mathrm{~m}$ entre une zone urbanisée (puits perdus, décharges) et une zone de captage.

\section{- Propagation de la pollution à l'aval d'une décharge.}

Les apports de cette étude à l'aval d'une décharge communale partiellement en eau à l'aide d'une vingtaine de points d'observation et près de 18 mois d'observation, sont les suivants :

- la partition de la décharge en deux zones distinctes permet de confirmer que les pollutions induites par des ordures ménagères et par des déchets de construction peuvent être qualifiées respectivement de chlonurée sodique et de sulfatée calcique;

- on observe nettement une zone de réduction à l'aval immédiat de la décharge d'ordures ménagères, qui se manifeste par un abaissement spectaculaire de la teneur en nitrates, compensé par des apparitions de nitrites et d'ammonium; les teneurs en azote total sont relativement plus faibles qu'en amont, ce qui laisse supposer un dégagement d'azote élémentaire;

- les teneurs en ions $\mathrm{Ca}$ et $\mathrm{CO}_{3} \mathrm{H}$ sont dans cette zone plus faibles que le laisseraient prévoir les apports polluants. La teneur en $\mathrm{Ca}$ baisse en effet légèrement, celle en $\mathrm{CO}_{3} \mathrm{H}$ augmente peu et les teneurs en $\mathrm{Cl}, \mathrm{Na}$,
$K$ augmentent très fortement, alors que ces derniers ions dans les apports sont en quantité inférieure ou égale à celle des premiers;

- par voie de conséquence, les meilleurs traceurs de la pollution sont dans l'ordre d'intérêt: $\mathrm{NH}_{4}, \mathrm{~K}, \mathrm{Na}, \mathrm{Cl}$;

- l'extension de la zone polluée organiquement à l'aval de la décharge serait d'environ $300 \mathrm{~m}$ et celle influencée d'une façon significative par les sels dissous serait de 1 à $2 \mathrm{~km}$ pour le $\mathrm{Cl}$ et $\mathrm{Na}$ et $500 \mathrm{~m}$ pour $\mathrm{K}$;

- nous avons pu constater, à l'aval de la zone en eau, des teneurs en $\mathrm{Cl}, \mathrm{Na}$ et $\mathrm{K}$ trois à cinq fois plus faibles qu'ailleurs, celles de $\mathrm{NH}_{4}$ étant dix fois plus faibles qu'en aval de la zone sèche. Un phénomène local d'aération et de dilution en eau libre intervient peut-être;

- l'observation de la propagation d'une pollution massive et de courte durée de la décharge par des platras, a permis de calculer la vitesse locale de la nappe: $0,6 \mathrm{~m} /$ jour (ce qui correspond à une perméabilité du même ordre de grandeur que celle calculée par essai de pompage).

- Propagation de la pollution dans une nappe à partir d'un cours d'eau.

Les conclusions de l'étude sur la contamination d'une zone de captage à partir d'un cours d'eau sont les suivantes:

La vitesse de propagation de la pollution chimique $\left(\mathrm{NO}_{3}\right)$ dans la nappe alluviale depuis le Vieux-Rhône jusqu'aux puits en exploitation des zones de captage de Charmy et Crépieux est relativement rapide: 5 et $7 \mathrm{~h}$ environ pour 125 à $185 \mathrm{~m}$.

Les vitesses de propagation sont ralenties par le colmatage des berges et du terrain dû à la pollution physique des eaux de surface et ce, d'autant plus que la perméabilité de l'aquifère est faible. Au-dessus d'une perméabilité de $10^{-2} \mathrm{~m} / \mathrm{s}$ environ, ce phénomène ne semble plus déterminant.

La pollution physique et organique des eaux de surface atténue sensiblement la propagation de la pollution bactériologique en filtrant cette dernière au niveau des berges.

Lors de la vidange de Verbois 1972, le meilleur traceur chimique observé a été $\mathrm{NO}_{3}$, l'oxydabilité n'étant pas suffisamment précise et $\mathrm{NO}_{2}$ et $\mathrm{NH}_{4}$ ne passant pas ou très peu dans les puits. Le meilleur traceur bactériologique a été obtenu avec les coliformes, les germes totaux étant trop nombreux et insuffisamment spécifiques (et les Escherichia Coli étant trop facilement filtrés).

\section{- L'influence de la Rize sur la propagation de la pollution.}

Dans la zone étudiée, les infiltrations de la Rize ne sont pas suffisantes pour jouer le rôle de barrière hydraulique en infléchissant localement la piézométrie de façon sensible.

Les infiltrations de la Rize existent cependant. Une pollution accidentelle de ce ruisseau (d'autant plus sujette à conséquences que son débit est faible : 80 à $1301 / \mathrm{s}$ de septembre 1967 à novembre 1968) passerait en partie dans la nappe sous-jacente.

Toute la zone étudiée est sous l'influence des infiltrations protectrices du canal de Jonage (déjà mise en évidence) dont les eaux peu chargées atténuent par mélange la pollution venue du couloir de Decines. 


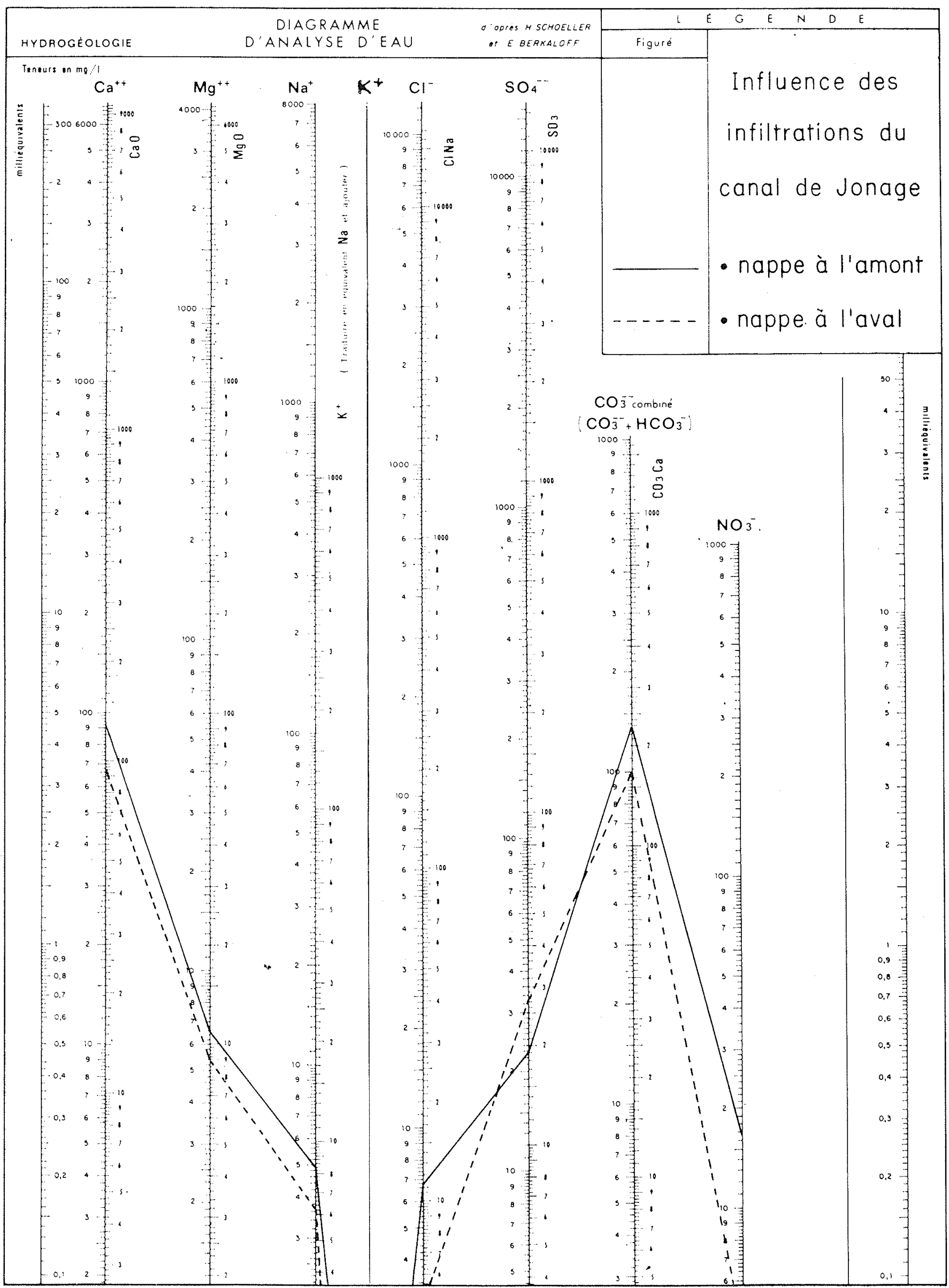




\section{Recherches expérimentales en zone non saturée}

Des essais de propagation ont consisté à simuler des rejets permanents en les marquant d'une injection instantanée de traceur (brome 82, iode 131, iodure de sodium, uranine, rhodamine)

Les injections ont été faites à proximité d'un sondage classique, puis au centre d'un dispositif circulaire de 7 piézomètres et enfin au voisinage de 3 forages cimentés à l'extrados sur toute la hauteur non saturée. Les souilles d'injection pénétraient jusqu'au mur de la couche de limons superficiels pour simuler au maximum les conditions habituelles de rejets (puisards). Toutefois, un essai a été fait par épandage en surface. Les débits d'eau injectés ont varié entre 0,2 et $2,6 \mathrm{~m}^{3} / \mathrm{h}$.

Les mesures ont porté sur la distribution du temps de séjour entre le point d'injection et la nappe sollicitée localement par un pompage dans le point d'eau testé.

La plus grande profondeur étudiée a été d'une quinzaine de mètres.

La pollution artificielle s'est propagée jusqu'à la nappe avec une vitesse d' $1 \mathrm{~m} / \mathrm{h}$ environ, la vitesse à travers les limons superficiels lors de l'essai d'épandage étant dix fois plus faible.

\section{Recherches expérimentales en zone saturée}

Des expérimentations préliminaires ayant comporté des mesures d'activité en radon, en tritium, en carbone 14, un traçage en puits unique avec du brome 82 , une étude de la diffusion d'une saumure (CINa) injectée dans la nappe et suivie par des mesures de conductivité au sol, enfin des injections et prélèvements de traceurs chimiques ou radioactifs dans un système multipuits, ont permis :

- de mettre en évidence et de localiser sur un site expérimental les couches de perméabilité différente;

- d'apprécier la vitesse relativement élevée de la nappe dans ce site : 4 à $5 \mathrm{~m} /$ jour;

- de mettre au point une méthode de détermination du sens d'écoulement et de la zone contaminée par un polluant en utilisant un puits unique.

Cette méthode consiste à mesurer la conductivité électrique du sol pour suivre la propagation d'une solution concentrée de Chlorure de sodium injectée sur toute la hauteur de l'aquifère. La mesure permet d'apprécier la concentration en sels dans la zone contaminée.

La méthode ayant été mise au point par le Centre d'Information Géologique de l'Ecole Nationale Supérieure des Mines de Paris, avec la collaboration du Service Géologique d'Alsace-Lorraine, les mêmes organismes l'ont mise en œuvre pour procéder à une étude de la dispersion des pol luants à l'état dissous.
Quatre sites ont été étudiés de cette façon.

Dans tous les cas, outre le calcul des coefficients intrinsèques de dispersion longitudinale et transversale, il a été possible de confirmer la direction de propagation de la pollution et de mesurer les vitesses moyennes de son parcours: une dizaine de mètres par jour ( 7 à $13 \mathrm{~m}$ ).

En outre, sur un cinquième site, constitué par une décharge communale, la géophysique a tenté de travailler sur les sels minéraux émis dans la nappe par cette décharge. Les résultats obtenus n'ont pas permis de calculer les coefficients de dispersion. Toutefois, ceux-ci ont pu être obtenus à partir des mesures de résistivité effectuées sur une ligne de piézomètres recoupant la langue polluée.

\section{Conclusion}

Les études effectuées sur la plaine de Lyon ont apporté une connaissance détaillée de la qualité des eaux et des menaces qui pèsent sur cette qualité. Elles nous ont permis de mettre au point une méthode d'observation systématique et de contrôle des nappes souterraines.

Les recherches sur la propagation et la dispersion des polluants ont eu un aspect plus méthodologique encore. Elles semblent avoir apporté le moyen de construire un modèle mathématique relativement élaboré, permettant de répondre à la question suivante: une masse connue de polluant étant injectée en un endroit donné, quelle sera la loi d'évolution de la concentration en ce même polluant, en tous points de la nappe? Il ne peut s'agir, certes, que d'une réponse très approximative, mais capable cependant d'aider à la définition des périmètres de protection des captages d'eaux souterraines.

\section{Bibliographie}

Busse ( $\mathrm{J}-\mathrm{F}$ ), Grejzes (C) et LACrolx ( J - L ) - La pollution et sa propagation dans la nappe alluviale de l'Est lyonnais». Deuxieme colloque sur la pollution et la protection des eaux de la région Rhône-Alpes (Lyon 5-6-7 décembre 1972).

Busse (J.-F.), Calmels (P.) et Molinari (J.). - «Propagation de la pothition entre le sol et la nappe (zone non saturée) dans la plaine de l'Est lyonnais ». Deuxième colloque sur la pollution et la protection des eaux de la région Rhône-Alpes (Lyon, 5-6-7 décembre 1972).

David (L.). - « Formations g'aciaires et fluvio-glaciaires de la région lyonnaise ». Doc. Labo. Géol. Fac. Sc. Lyon, n 22 (1967).

FRIED (J.-J.). — «Etudes théoriques et méthodologiques de la dispersion en milieu poreux naturel ». Thèse doctorat d'Etat ès-Sciences physiques. Faculté des Sciences de l'Université de Bordeaux (1972). 


\section{Discussion}

Président : M. J. MARGAT

M. le Président remercie M. Busse pour son exposé fort bien illustré et ouvre la discussion.

Que's sont les rapports entre les vitesses de progression des pollutions, d'une part, d'après l'expérience, et d'autre part, d'après le calcul, en supposant que le terrain est homogène avec une porosité de $35 \%$, demande M. de Cazenove (Soletanche)?

M. Busse répond :

Avec une porosité de $35 \%$, ce rapport est de 2 à 3 en moyenne; il peut atteindre 10 lors des mesures in situ avec traceurs radioactifs. Le rapport de 2 à 3 est obtenu avec la méthode géophysique sur saumure; il s'agit là d'une méthode moyenne, où l'injection de saumure est faite sur toute !a hauteur aquifère. Au contraire, pour caler le modèle, il a fallu mettre en place des zones avec des perméabilités très fnement réparties; le modèle n'a pu donner de bons résultats qu'après intercalation des couches de très forte perméabilité.

Sur une question de $M$. le Président, M. Busse indique :

La méthode géophysique a été choisie après avoir mis en concurrence plusieurs méthodes et, en particulier, la méthode classique consistant à injecter un traceur naturel ou radioactif en un point et à le rechercher dans une batterie de piézométres disposés en arc de cercle à l'aval. Cette méthode a été appliquée sur le même site. On a injecté de la saumure dans le puits et on a recherché le paramètre de diffusion de la saumure à l'aval; il coincidait très correctement avec ce qu'avait indiqué les traceurs, à cette nuance près que les traceurs donnaient localement des «pointes 》 beaucoup plus fortes; ainsi on obtenait une vitesse moyenne de 5 à $7 \mathrm{~m}$ /jour par la méthode géophysique sur saumure; suivant l'axe principal, ces mêmes vitesses, montaient jusqu'à $10 \mathrm{~m} /$ jour avec les traceurs, mais, par endroits, les indications de certains piézomètres conduisaient à des vitesses de $20 \mathrm{~m} / \mathrm{jour}$, parce que ces piézomètres étaient intéressés par les couches les plus conductrices.

M. Gleizes (Agence Rhône-Méditerranée-Corse) précise

Les études de dispersion, évoquées par M. Busse, ont été dirigées par MM. EMSEllem et Fried (Centre d'Informatique Géologique de l'Ecole Nationale Supérieure des Mines de Paris).

La méthode géophysique a été retenue, pour la détermination des coefficients intrinsèques de dispersion longitudinale et transversale, à partir d'un modèle mathématique prenant en considération les différentes couches et restituant les résultats de la mesure électrique g'obale.

M. le Président clôt la discussion et tire, en ces termes, la conclusion de la séance :

Je crois qu'on peut retenir de ces trois exposés que l'hydrogéologie commence à démontrer ses capacités d'aborder les problèmes assez complexes touchant à la gestion et de servir de base à des évaluations facilitant des choix économiques; ce n'est pas si ancien en fin de compte. Et, surtout, c'est aussi une bonne démonstration de la très grande diversité des méthodes mises en jeu, de la variété des organismes et des personnes qui contribuent à ces travaux. L'hydrogéologie est dans ses applications une technique multidisciplinaire. 